\title{
Detection of Defects in Glass Sheet using C.S.C based Segmentation Method
}

\author{
Tribhuwan Singh \\ M.Tech (CSP) \\ student, \\ JNU, Jaipur, India
}

\author{
Rajeshwar Lal \\ Dua \\ Professor, JNU, \\ Jaipur, India
}

\author{
Sunil Agrawal \\ Asst. Professor, \\ Panjab \\ University, \\ Chandigarh
}

\author{
Abhishek \\ Acharya \\ Asst. Professor, \\ Govt. Engineering \\ College \\ Bikaner (Rajasthan)
}

\begin{abstract}
Manual detection of defects in large size glass sheets is an extremely tedious process. The manual inspection is a slow process, time consuming and prone to human error and gives wretched quality glass sheet. It is a major reason of abashment for manufacturers. Thus automatic inspection offer manufacturers an opportunity to improve quality and reduce cost and overcome many disadvantages.

In this paper author suggest C.S.C based Segmentation for defect detection in glass sheet. These defect include Line, Edge and Scratch etc. The above proposed scheme is based on Subjective scale Analysis. Further Conversion Time Analysis is also used. For absolute Visibility of defect in Glass sheet C.S.C is useful tool. The proposed Segmentation method is Splendid approach to avoid false Edge detection.
\end{abstract}

Keywords: Edge Detection, Segmentation, C.S.C, Defect.

\section{INTRODUCTION}

In the past human vision has played a primary role in quality inspection. The essence concept is most vital aspect for glass manufacturers. The glass industry has had no well-established organization to support it in research and defend its viability, unlike other industries. Today it is undergoing dramatic changes. As in other mature industries, resisted change in its tried-and-true manufacturing processes [1].Products of the right quality have to be delivered at short notice, within strictly defined time windows and in the requested volume [2]. The revolutionary power to bring best quality products to the market quickly is becoming a necessity for industries to improve or even maintain their market position.

In order to determine the true quality of the glass, correct classification of the detected defects is very important. But, the classification can only be as good as the quality and significance of the initial defect image detected by the inspection system [3].

The rest of the paper is organized as follows: Section two discuss various defects. Section three briefly described detection of defects. Section four introduce color space conversion test using visibility test for different color space and segmentation method using edge detection technique.

\section{TYPE OF DEFECTS}

Defects must be avoided at all cost. So considering this a glass sheet is before manufacturing sent to defect detection unit for testing and valediction of defect. The different types of defect present in glass are:
2.1. Point Defects: These are inclusion trapped in glass as a defect during its production. Example: Stone, Bubble etc.

2.2. Surface defects: These defect cause major problem for manufacturer, when the process include surface treatment stage. Example: Hole \& Spot.

2.3. Edge Defects: These defects are the main cause of breakage of glass sheet during production. It can be prevented by detecting at early stage. Example: Chips.

2.4. Line Defects: These are the irregular patches on surface of glass sheet. These can occur during Edge grinding and Corner cutting. These can be light or deep. Example: Scratches and Cracks.

2.5. Blemish Defects: These defect areas fairly large and relatively dark or bright regions [4] against background. Example: Water strains \& Low contrast defects.

2.6. Molecular Defects: This defect has an unmelted material embedded in glass sheet. This defect has the appearance of a lump. Example: Dust particles and Chemical residues.

\section{RELATED WORK}

In the field of defect detection in glass sheet considerable research utilizing in-line automated inspection system. Zhang, Hongxi [5], in their paper proposed a defect detection algorithm based on Discrete Fourier Transform (DFT) and optimal threshold method, mainly aiming at detecting bubble, stone, and crack of glass. The results demonstrate the proposed detection algorithm can detect and localize the defect region with high accuracy and better practicability. For low resolution images Zhao, Jie [6] proposed a method of canny edge detection, and thus the smallest connected region (rectangle) can be found. Finally, the AdaBoost method is adopted for classification. Experiments with 800 bubble images and 240 non-bubble images prove that the proposed method is effective and efficient for recognition of glass defects, such as bubbles and inclusions. A system [7] was designed to reproduce the real issues of an in-line quality control system which included three subsystems: an array of several CMOS cameras, a controllable roller conveyor, and a PC-based image processing system that is also responsible for the control of the other subsystems. The detection of the defects was performed by means of canny edge detection, with thresholds chosen according to some statistics of the images being processed. To detect the low-contrast regions on glass, a highly robust estimator, known as the Model-Fitting (MF) estimator [8] was 
developed by X. Zhuang et al. which used a modified MF estimator to robustly estimate the background model and as a by-product to segment the blemish defects, the outliers. The illumination irregularity was made as a parabolic function; the center area was made brighter than the perimeter of the image. A zero mean Gaussian noise was added to the ground truth and the amount of noise, the standard deviation of the Gaussian noise, and the depth of circle of the ground truth are controlled for each simulation.

\section{DEFECT DETECTION PROCESS}

Defects are Troublesome and irresolute. Furthermore appearing areas, defects can be detached into different section. There are three main steps for this process. Which are image processing module, C.S.C module and image segmentation module? The first step involves editing of image in database for best result in later stage. In next step C.S.C is used for best shows defect in image using proper color space. Using proper color space we can reduce the complexities during its detection in segmentation step.

\subsection{Color Space Conversion}

For appropriate result of segmentation process, it is necessary to adopt a appropriate color space. During image acquisition every image is in RGB format. So we have to convert RGB image in four other color spaces for better result. These are:

\subsubsection{RGB:}

RGB color space is really a way of representing images using three values Red, Green, and Blue, that will fool us into thinking we are seeing something in the real world. Images can thus be stored as a 3 arrays values, with each of the three values forming a single pixel, or point of color, to be displayed.

\subsubsection{HSV:}

Hue, Saturation, Value defines a type of color space. The HSV color space has three components: hue, saturation, value [9]. 'Value' is sometimes substituted with 'brightness' and then it is known as HSB. In HSV, hue represents color. In this model hue is an angle from 0 degree to 360 degree. Saturation indicates the range of grey in color space. It ranges from 0 to $100 \%$. Sometimes the value is calculated from 0 to 1 . When the value is ' 0 ', the color is grey and when the value is ' 1 ', the color is a primary color. Value is the brightness of the color and varies with color saturation.

\subsubsection{YCbCr:}

This color space represents each color with 3 numbers, similarly as the RGB space. The Y component represents the intensity of the light. The $\mathrm{Cb}$ and $\mathrm{Cr}$ components indicate the intensities [10] of the blue and red components relative to the green component.

\subsubsection{NTSC:}

The NTSC color space is used in televisions in the United States. One of the main advantages of this format is that grayscale information is separated from color data, so the same signal can be used for both color and black and white sets [11]. In the NTSC format, image data consists of three components: luminance(Y), hue(I), saturation(Q). The first component, luminance, represents greyscale information, while the last two components make up chrominance (color information).

\subsubsection{Gray scale:}

Gray scale is a range of shades of gray without apparent color. The darkest possible shade is black, which is the total absence of transmitted or reflected light. The lightest possible shade is white, the total transmission or reflection of light at all visible wavelength s. Intermediate shades of gray are represented equal brightness levels of the three primary colors (red, green and blue) for transmitted light, or equal amounts of the three primary pigments (cyan, magenta and yellow) for reflected light.

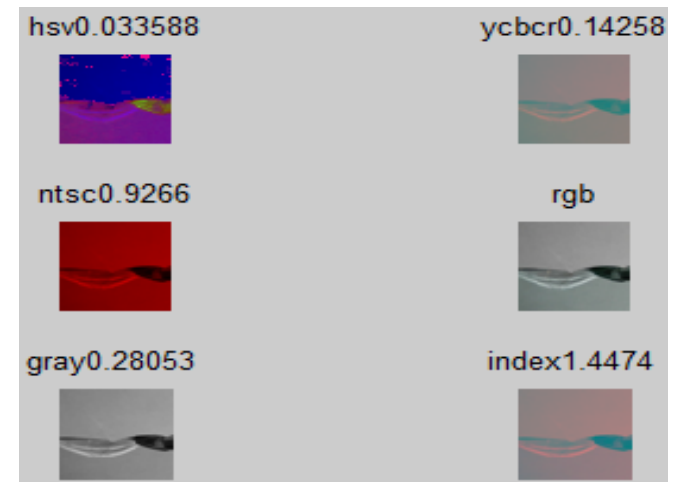

Figure 1: CSC Analysis

Once the conversion is complete, each color space is assigned a level from 1 to 5, known as Subjective Scale, according to the visibility of human visual perception. Highest degree of visibility of the defect in color space is marked by 5 and least degree of visibility is assigned by 1 . The conversion time of each image is calculated above from RGB to other color space.

\subsection{Image Segmentation}

Segmentation subdivides an image into its constituent's objects. The detail of subdivision is carried depends on the problem being solved. Segmentation should stop when the region of interest in an application have been detected. The quality of segmentation effect influences the follow-up analysis of the images directly [12]. Therefore accurate segmentation of image is very essential. Segmentation algorithm for monochrome images generally based on one of two basic categories dealing with properties of intensity values: discontinuity and similarity. In the first category, boundaries of region [11] are sufficiently different from each other. Edge based Segmentation is the principal approach used in this category. Region based Segmentation approaches in the second category based on partitioning an image into regions that are similar according to a set of predefined criteria.

Edge Detection is the approach used most frequently for segmenting images based on local changes in intensity. There are three fundamental steps for edge detection which are Image smoothing for noise reduction, Detection of edge point and Edge localization. For finding the edge strength and direction at location $(\mathrm{x}, \mathrm{y})$ of an image, $\mathrm{f}$, is the gradient, denoted by $\nabla f$, and defined as the vector

$$
\nabla f \equiv \operatorname{grad}(f) \equiv\left(\begin{array}{l}
g_{x} \\
g_{y}
\end{array}\right)=\left(\begin{array}{l}
\frac{\partial f}{\partial x} \\
\frac{\partial f}{\partial y}
\end{array}\right)
$$

The magnitude of vector $\nabla f$, denoted as $\mathrm{M}(\mathrm{x}, \mathrm{y})$, where

$$
\mathrm{M}(\mathrm{x}, \mathrm{y})=\operatorname{mag}(\nabla f)=\sqrt{g_{x}^{2}+g_{y}^{2}}
$$

The direction of gradient vector is given by the angle 


$$
\alpha(x, y)=\tan ^{-1}\left(g_{y} \mid g_{x}\right)
$$

Gradient operator obtaining the gradient of animage require computing the partial derivatives $\frac{\partial f}{\partial x}$ and $\frac{\partial f}{\partial y}$ at every pixel location in the image

$$
\begin{array}{r}
g_{x}=\frac{\partial f(x, y)}{\partial x}=f(x+1, y)-f(x, y)(\mathrm{d}) \\
g_{y}=\frac{\partial f(x, y)}{\partial y}=f(x, y+1)-f(x, y)(\mathrm{e})
\end{array}
$$

\begin{tabular}{|l|l|l|}
\hline$Z_{1}$ & $Z_{2}$ & $Z_{3}$ \\
\hline$Z_{4}$ & $Z_{5}$ & $Z_{6}$ \\
\hline$Z_{7}$ & $Z_{8}$ & $Z_{9}$ \\
\hline
\end{tabular}

Now formula for edge detection algorithm using technique used in this paper is

$$
\begin{aligned}
& g_{x}=\frac{\partial f}{\partial x}=\left(Z_{7}+2 Z_{8}+Z_{9}\right)-\left(Z_{1}+2 Z_{2}+Z_{3}\right) \\
& g_{y}=\frac{\partial f}{\partial y}=\left(Z_{3}+2 Z_{6}+Z_{9}\right)-\left(Z_{1}+2 Z_{4}+Z_{7}\right)
\end{aligned}
$$

\section{RESULTS}

The proposed method has been applied on digital glass sheets for finding various defects using MATLAB. Test has been conducted on 74 images having various defects that can be presented in glass sheet. Two factors taken into account for analysis in which one of the method is shown which is:

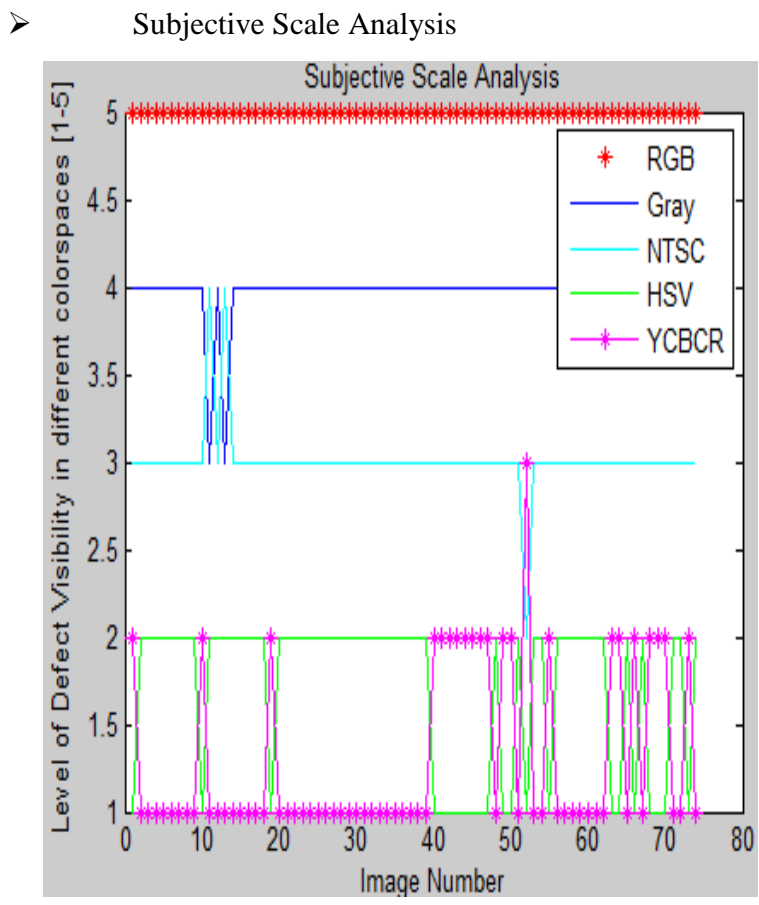

Figure 2: Subjective Scale Analysis

According to this figure different levels are assigned for different color space which is from 1 to 5 . As seen in figure level 5 is assigned for RGB and in decreasing order last 1 is assigned for $\mathrm{YCbCr}$.

So combining both the results, as discussed earlier in CSC Analysis and in SSA Gray color space conversion gives best result as compare to others.

Now RGB image obtained is then given as input image to segmentation algorithm implemented. The input image is read and converted into gray color map. Then with the help of Edge detection algorithm the final and complete result have been shown in figure 3 .

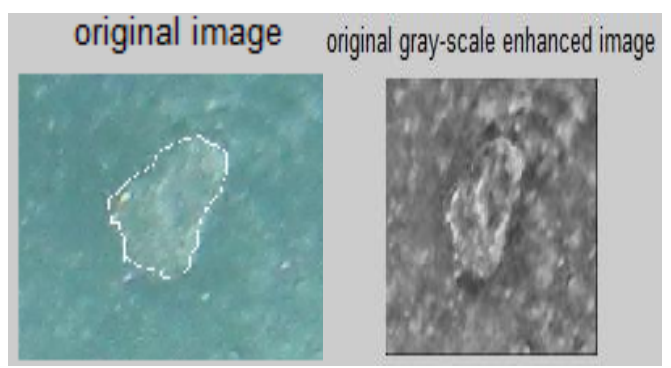

Figure 3(a)
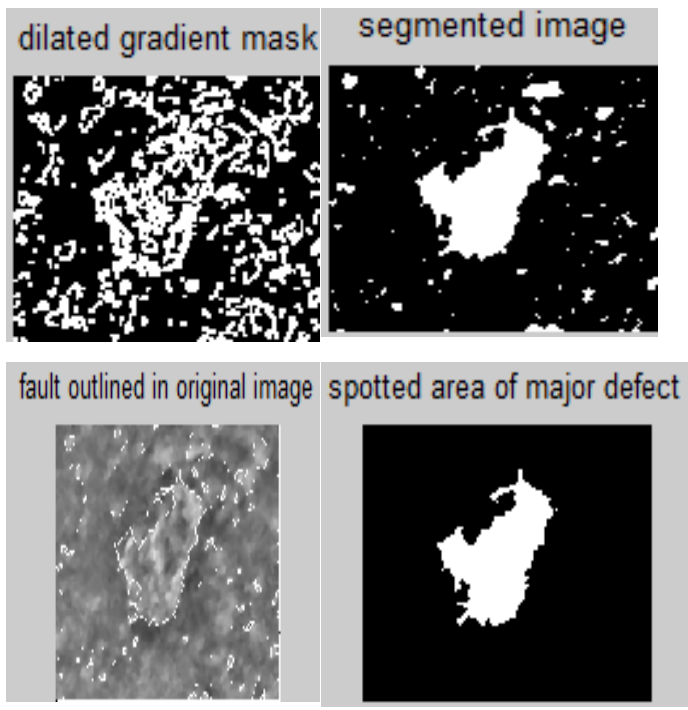

Figure 3(b)

Figure 3(a) and 3(b): Blemish Defect Detection

\section{CONCLUSION}

This paper briefly discussed various types of defects in glass sheet. Different type of color space techniques have been compared and analysed for their performance. On the above reported theory Gray scale color map is best for this technique. The segmentation algorithm based on Edge detection technique is often used in Medical imaging.Our future work will focus on: 1) Use more appropriate and automated resources to improve the efficiency of defect detection technology. 2) Implement the algorithm on bulk of defective glass sheets.

\section{REFERENCES}

[1] E.N. Malamas, E.G.M. Petrakis, M. Zervakis, L. Petit, J.D. Legat, "A survey on industrial vision systems, applications and tools, Image and Vision Computing", 2003, pp. 171-188.

[2] F. Adamo, F. Attivissimo, A. Di Nisio, M. Savino, "An Automated visual inspection system for the glass industry", In: Proc. Of 16th IMEKO TC4 Symposium, Florence, Italy, Sept 2008, pp. 22-24. 
[3] M.A.Coulthard, "Image Processing for Automatic Surface Defect Detection," Surface Inspection Ltd, UK, pp.192-196.

[4] Nishu Gupta, Sunil Agrawal, "Glass Defect Detection Techniques using Digital Image Processing -A Review", IJCA Special Issue on IP Multimedia Communications, October 2011, pp. 65-67.

[5] Zhang, Hongxi, Guo, Zhenduo, Qi, Zegang, Wang, Jiuge "Research of glass Defect Detection based on DFT and optimal threshold Method" CSIP, 2012 International Conference on 24-26 Aug. 2012 Pages: 1044-1047.

[6] Jie Zhao, Xu Zhao and Yuncai Liu, "A Method for Detection and Classification of Glass Defects in Low Resolution Images," Sixth International Conference on Image and Graphics, 2011, pp.642-647.

[7] Francesco Adamo and Mario Savino, "A low-cost Inspection system for online defects assessment in satin glass," 2009, pp. 1304-1311.
[8] Chang-Hwan Oh, HyonamJoo and Keun-HoRew, "Detecting Low-Contrast Defect Regions on Glasses Using Highly Robust Model-Fitting Estimator," International Conference on Control, Automation and Systems, 2007, pp. 2138-2141.

[9] Li Shuhua, Guo Gaizhi, "The application of improved HSV color space model in image processing", 2nd International Conference on Future Computer and Communication, 2010.

[10] Wang Yuedong, XueHeru, "Studies on Color Space Selection and Methods of Segmentation Quality Evaluation", 1st International Conference on Information Science and Engineering, 2009.

[11] Digital Image Processing Using MATLAB by Rafael C. Gonzalez and Richard Woods, second edition.

[12] Wang Yuedong, XueHeru, "Studies on Color Space Selection and Methods of Segmentation Quality Evaluation", 1st International Conference on Information Science and Engineering, 2009. 